\title{
Éducation thérapeutique du patient en médecine physique et de réadaptation, une dynamique en marche
}

\author{
Patient therapeutic education and physical and rehabilitation medicine, the best is coming!
}

\author{
E. Coudeyre $\cdot$ S. Bendaya \\ C Springer-Verlag France 2013
}

L'OMS Europe a défini l'éducation thérapeutique (ETP) qui a pour objet de «former le malade pour qu'il puisse acquérir un savoir-faire adéquat, afin d'arriver à un équilibre entre sa vie et le contrôle optimal de sa maladie. L'ETP du patient est un processus continu qui fait partie intégrante des soins médicaux. L'ETP du patient comprend la sensibilisation, l'information, l'apprentissage, le support psychosocial, tous liés à la maladie et au traitement. La formation doit aussi permettre au malade et à sa famille de mieux collaborer avec les soignants ». Ainsi, l'ETP des patients contribue pour les malades chroniques à une meilleure connaissance de leur pathologie et de sa prise en charge, les faisant ainsi devenir acteur de leur propre santé. L'objectif principal de l'ETP est l'amélioration de la prise en charge de la pathologie via une diminution de la morbidité ou une réduction de la survenue de certaines complications ou incidents. L'ETP a pour finalité l'acquisition par le patient de compétences d'autosoins et d'adaptation. Parmi les compétences d'autosoins, certaines sont primordiales dans la prise en charge des patients en MPR, comme la réalisation de gestes techniques (autosondage pour les vessies neurologiques), la mise en œuvre de modification du mode de vie (équilibre diététique et exercice) pour les pathologies cardiovasculaires, l'implication de l'entourage dans la gestion de la maladie et des traitements (place de l'aidant pour les pathologies neurologiques).

Les circulaires d'avril 2008 inscrivent l'éducation thérapeutique dans les missions des services de soins de suite et de réadaptation (SSR) au même titre que les soins, la rééducation et la réadaptation, l'accompagnement à la réinsertion. Ainsi, le catalogue spécifique des actes de rééducation et de réadaptation (CSARR) référence près d'une trentaine d'actes permettant de mieux valoriser les activités d'information et d'éducation des patients. La spécificité de la prise en charge en MPR permet de ne pas se restreindre à des stratégies éducatives isolées dont l'impact peut être limité mais de pouvoir $\mathrm{y}$ associer des techniques de rééducation et de réadaptation.

Cette monographie consacrée à l'éducation thérapeutique du patient est riche par la diversité de son contenu. Trois articles permettent de faire le point sur l'état de l'art à partir des données de la littérature dans le domaine de l'arthrose (ETP avant arthoplastie totale de genou) ou des pathologies cardiovasculaires (optimisation de l'alliance thérapeutique autour de l'activité physique dans les maladies cardiovasculaires) et de travaux originaux (évaluation des freins à la pratique de l'activité physique chez les patients porteurs d'une sclérose en plaques). Les autres articles permettent le partage d'expérience de programmes d'ETP validés dans le champ des pathologies neurologiques concernant autant les accidents vasculaires cérébraux (prévention des chutes après un AVC) que la maladie de Parkinson (mise en œuvre d'un programme pour patient parkinsonien à la phase expérimentale) ou les lésés médullaires (expériences éducatives dans le champ de l'escarre). Enfin, un dernier article présente la méthodologie d'élaboration d'un guide d'aide à la mise en place d'un programme pour la lombalgie chronique (principes méthodologiques de l'élaboration d'un guide destiné aux équipes).

En espérant que cette monographie réponde aux attentes des lecteurs et contribue à l'essor de l'ETP en MPR, nous vous souhaitons une bonne lecture.

\author{
E. Coudeyre $(\triangle) \cdot S$. Bendaya \\ Service de médecine physique et de réadaptation, $\mathrm{CHU}$ \\ Clermont-Ferrand, Hôpital Nord, 61 rue de Chateaugay, BP \\ 30056, F-63118 Cébazat \\ e-mail : ecoudeyre@chu-clermontferrand.fr
}

Hôpital Rothschild, service médecine physique et de réadaptation fonctionnelle, 5, rue Santerre, F-75012 Paris 